\title{
Получение и исследование термоэлектрических свойств сплавов Гейслера $\mathrm{Fe}_{2} \mathrm{TiSn}_{1-x} \mathbf{S i}_{x}$
}

\author{
(С) А.И. Воронин ${ }^{1,2}$, В.Ю. Зуева ${ }^{1}$, Д.Ю. Карпенков ${ }^{1}$, \\ Д.О. Московских ${ }^{1}$, А.П. Новицкий ${ }^{1}$, Х. Мики ${ }^{3}$, В.В. Ховайло ${ }^{1}$ \\ ${ }^{1}$ Национальный исследовательский технологический университет „МИСиС“, \\ 119049 Москва, Россия \\ 2 Южно-Уральский государственный университет (национальный исследовательский университет), \\ 454080 Челябинск, Россия \\ ${ }^{3}$ Frontier Research Institute for Interdisciplinary Science, Tohoku University, \\ 980-8578 Sendai, Japan \\ E-mail: voronin@misis.ru
}

(Получена 27 декабря 2016 г. Принята к печати 12 января 2017 г.)

Проведены экспериментальные исследования поликристаллических сплавов $\mathrm{Fe}_{2} \operatorname{TiSn}_{1-x} \mathrm{Si}_{x}(0 \leq x \leq 1)$, которые теоретически предсказаны как высокоэффективные термоэлектрические материалы. Структурные исследования показали, что частичное замещение $\mathrm{Sn}$ на $\mathrm{Si}$ приводит к формированию многофазного состояния в образцах с $x>0$. Примесные фазы приводят в целом к существенному понижению коэффициента Зеебека и повышению теплопроводности образцов $\mathrm{Fe}_{2} \mathrm{TiSn}_{1-x} \mathrm{Si}_{x}$, что не позволяет рассматривать эти материалы в качестве перспективных термоэлектриков.

DOI: $10.21883 /$ FTP.2017.07.44645.31

\section{1. Введение}

Термоэлектрические материалы способны напрямую преобразовывать тепловую энергию в электрическую, что может быть эффективно применено в создании устройств утилизации бросового тепла и альтернативной энергетики. Сплавы Гейслера вызывают значительный интерес как низко- и среднетемпературные термоэлектрические материалы на протяжении последних двадцати лет. Так называемые полные сплавы Гейслера определяются как тройные интерметаллические соединения с химической формулой $X_{2} Y Z$, где $X$ и $Y-$ как правило $3 d$ - и $4 d$-переходные элементы, а $Z$ - металлоид. Они имеют объемноцентрированную кубическую кристаллическую структуру (структурный тип $L 2_{1}$ ), где атомы элемента $X$ занимают позиции в центре куба, а атомы элементов $Y$ и $Z$, поочередно, занимают угловые позиции (рис. 1) [1,2]. С точки зрения термоэлектрических свойств наиболее изученным материалом такого типа является $\mathrm{Fe}_{2} \mathrm{VAl}$ [3-5], который хотя и демонстрирует высокие значения термоэдс, но тем не менее его термоэлектрическая добротность $Z T$ не превышает, как правило, 0.3 из-за относительно большой теплопроводности.

Наряду с $\mathrm{Fe}_{2} \mathrm{VAl}$ в последние годы отмечается растущее внимание к поиску и исследованию новых термоэлектрических материалов на основе полных сплавов Гейслера $X_{2} Y Z$ [6,7]. Экспериментальные исследования термоэлектрических свойств сплавов Гейслера $\mathrm{Fe}_{2} \mathrm{TiZ}$ $(Z=\mathrm{Al}, \mathrm{Sn})$ показали $[8,9]$, что коэффициент Зеебека в этих соединениях не превышает (по модулю) 50 мкВ/К. Кроме этого, большое количество дефектов упаковки, возникающих из-за атомного разупорядочения структурных подсистем Fе и Тi, приводит к появлению локаль- ных магнитоупорядоченных кластеров, что негативно сказывается на тепловых и транспортных свойствах [10]. В недавней работе [11] были проведены теоретические исследования сплавов Гейслера $\mathrm{Fe}_{2} \mathrm{TiSn}$ и $\mathrm{Fe}_{2} \mathrm{TiSi}$. Была рассчитана зонная структура этих сплавов методами из первых принципов и показана возможность достижения значения термоэдс до $-300 \mathrm{M \kappa} \mathrm{B} / \mathrm{K}$ при концентрации носителей заряда от $10^{20}$ до $10^{21} \mathrm{~cm}^{-3}$ при комнатной температуре. В результате авторами работы [11] был предложен способ реализации высокого значения термоэлектрической эффективности путем замещения олова на кремний и получения материалов состава $\mathrm{Fe}_{2} \mathrm{TiSn}_{1-x} \mathrm{Si}_{x}$. Для проверки теоретических предсказаний нами были изготовлены и исследованы поликристаллические образцы сплавов этих соединений.

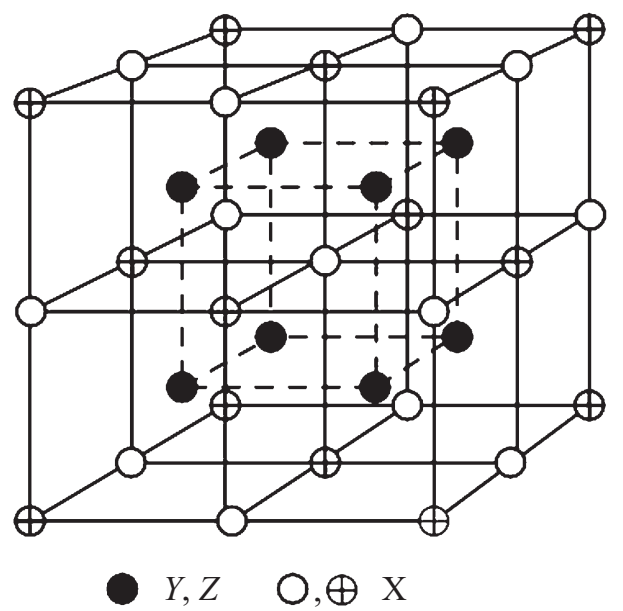

Рис. 1. Кристаллическая решетка сплава Гейслера $X_{2} Y Z$. 


\section{2. Образцы и методы исследования}

Исследования были проведены на образцах сплавов Гейслера состава $\mathrm{Fe}_{2} \mathrm{TiSn}_{1-x} \mathrm{Si}_{x}$, где $x$ изменялся от 0 до 1 с шагом 0.2. Синтез образцов проводился методом индукционной плавки на установке Indutherm MC 100V. В качестве исходных материалов использовались чистые элементы $\mathrm{Fe}, \mathrm{Ti}, \mathrm{Sn}$ и $\mathrm{Si}$. Исходные элементы помещались в открытый керамический тигель и подвергались высокочастотному индукционному нагреву в атмосфере аргона в течение 4 мин до полного расплавления компонентов. Полученные слитки подвергались гомогенизирующему отжигу в кварцевой ампуле в вакууме при температуре $973 \mathrm{~K}$ в течение 4 дней. Фазовый состав слитков определялся методом рентгеновской дифракции на дифрактометре Philips X'Pert с излучением Мо- $K_{\alpha}$. Теплопроводность, электросопротивление и коэффициент термоэдс поликристаллических образцов измерялись на установке измерения физических свойств (Physical Properties Measurements System, Quantum Design) при температуре от 20 до $350 \mathrm{~K}$.

\section{3. Результаты исследований}

На рис. 2 представлены дифрактограммы поликристаллических образцов составов $\mathrm{Fe}_{2} \mathrm{TiSn}_{1-x} \mathrm{Si}_{x}$ после индукционной плавки и отжига. Рентгеновские данные указывают на то, что в однофазном состоянии находится только сплав состава $\mathrm{Fe}_{2} \mathrm{TiSn}$. Частичное замещение $\mathrm{Sn}$ на $\mathrm{Si}$ приводит к появлению нескольких фаз в образцах $\mathrm{Fe}_{2} \mathrm{TiSn}_{1-x} \mathrm{Si}_{x}$ с $0<x<1$. Наличие примесной фазы в поликристаллическом образце $\mathrm{Fe}_{2} \mathrm{TiSi}(x=1)$ хорошо согласуется с литературными данными, которые свидетельствуют о том, что данный сплав может быть получен в однофазном состоянии только в виде тонких пленок [12]. Что касается образцов с частичным замещением $\mathrm{Sn}$ на $\mathrm{Si}$, то формирование примесных фаз в $\mathrm{Fe}_{2} \mathrm{TiSn}_{1-x} \mathrm{Si}_{x}$ обусловлено, по-видимому, специфическими особенностями фазовой диаграммы тройной системы $\mathrm{Fe}-\mathrm{Ti}-\mathrm{Sn}$. А именно исследования фазовых равновесий, проведенные в недавних работах $[13,14]$, указывают на узкую область гомогенности соединения $\mathrm{Fe}_{2} \mathrm{TiSn}$, которое становится нестабильным даже при малых отклонениях содержания $\mathrm{Sn}$ от стехиометрического.

Результаты измерений температурных зависимостей коэффициента Зеебека $S$, электрического сопротивления $\rho$ и теплопроводности $\kappa$ показаны на рис. $3-5$. Максимальное значение коэффициента Зеебека 31 мкВ/К наблюдается при комнатной температуре для состава $\mathrm{Fe}_{2} \mathrm{TiSn}$, что коррелирует с данными, полученными в работе [9], в то время как в остальных образцах $S$ было значительно ниже (рис. 3). Наблюдаемое понижение коэффициента Зеебека может быть объяснено появлением значительного количества примесных фаз интерметаллических соединений $\mathrm{Fe}_{2} \mathrm{Ti}$ и $\mathrm{FeSn}_{2}$ в сплавах
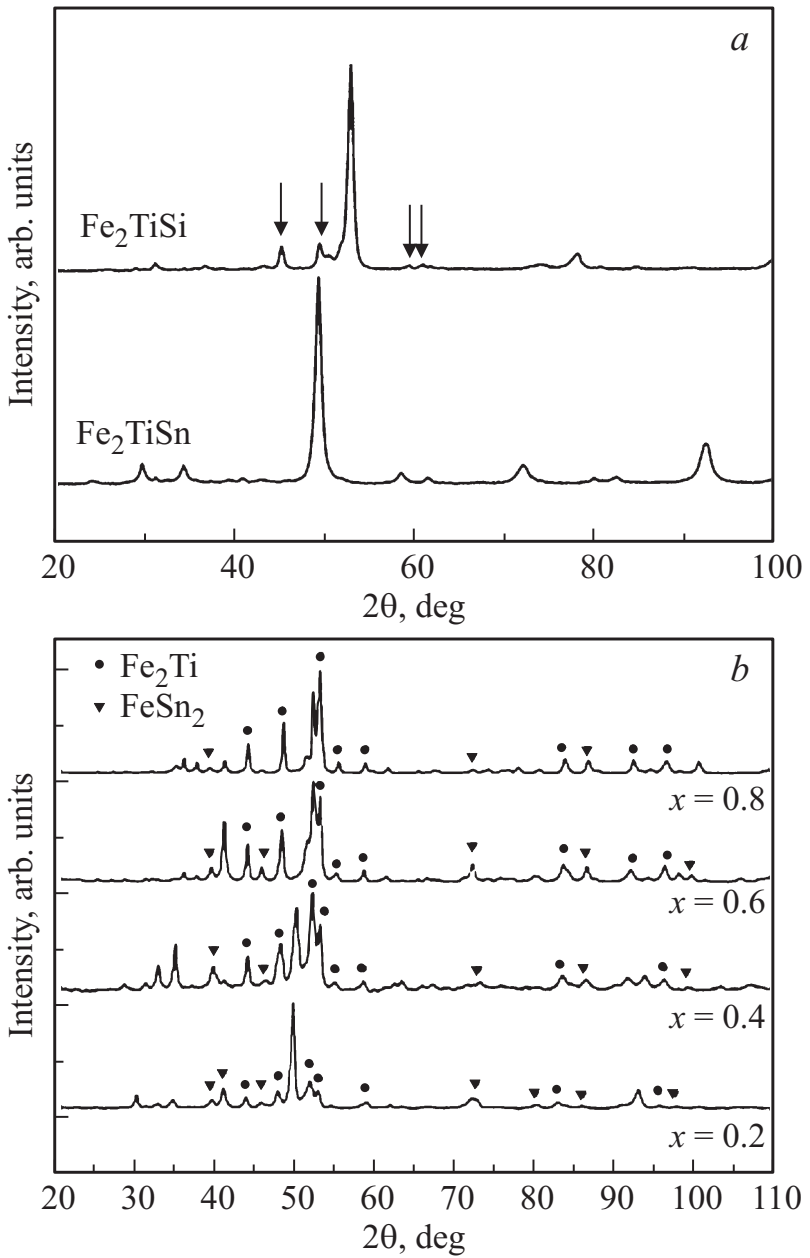

Рис. 2. Дифрактограмма отожженных образцов $\mathrm{Fe}_{2} \mathrm{TiSn}$, $\mathrm{Fe}_{2} \mathrm{TiSi}(a)$ и $\mathrm{Fe}_{2} \mathrm{TiSn}_{1-x} \mathrm{Si}_{x}(b)$.

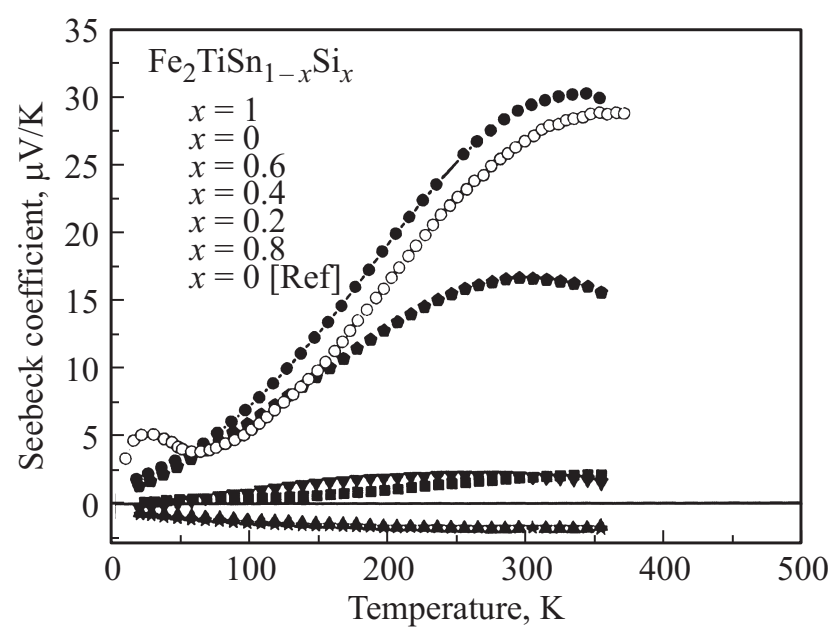

Рис. 3. Температурная зависимость коэффициента Зеебека поликристаллических образцов $\mathrm{Fe}_{2} \mathrm{TiSn}_{1-x} \mathrm{Si}_{x}$.

$\mathrm{Fe}_{2} \mathrm{TiSn}_{1-x} \mathrm{Si}_{x}$. Такое предположение согласуется в целом с данными по электрическому сопротивлению (рис. 4) и теплопроводности (рис. 5) сплавов $\mathrm{Fe}_{2} \mathrm{TiSn}_{1-x} \mathrm{Si}_{x}$, кото- 


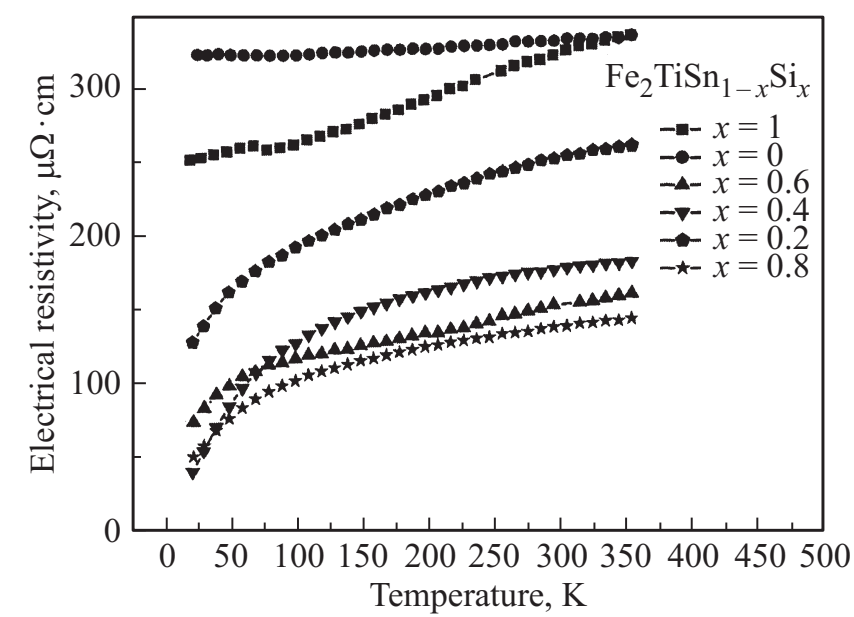

Рис. 4. Температурная зависимость электросопротивления поликристаллических образцов $\mathrm{Fe}_{2} \mathrm{TiSn}_{1-x} \mathrm{Si}_{x}$.

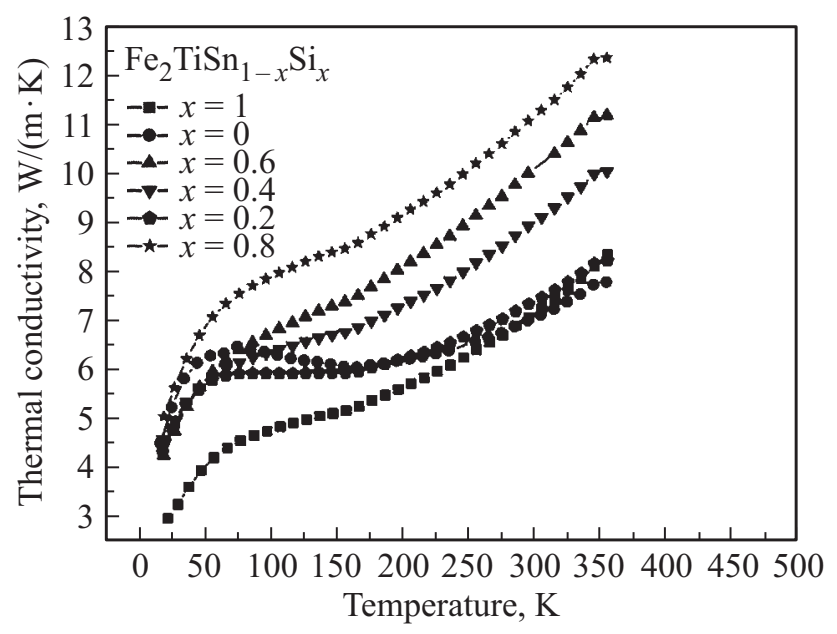

Рис. 5. Температурная зависимость теплопроводности поликристаллических образцов $\mathrm{Fe}_{2} \mathrm{TiSn}_{1-x} \mathrm{Si}_{x}$.

рые указывают на общую тенденцию к понижению $\rho$ и к повышению $\kappa$ в образцах с частичным замещением $\mathrm{Sn}$ на Si. Рассматривая данные для образца $\mathrm{Fe}_{2} \mathrm{TiSi}(x=1)$, можно отметить, что при температурах выше комнатной значения электрического сопротивления и теплопроводности в этом образце близки к соответствующим значениям в образце $\mathrm{Fe}_{2} \mathrm{TiSn}(x=0)$. Несмотря на то что содержание примесных фаз в $\mathrm{Fe}_{2} \mathrm{TiSi}$ является наименьшим среди всех исследуемых образцов $\mathrm{Fe}_{2} \mathrm{TiSn}_{1-x} \mathrm{Si}_{x}$ $(0<x<1)$ с многофазной структурой (рис. 2), на основании наших данных трудно утверждать, что полученные для исследуемого образца $\mathrm{Fe}_{2} \mathrm{TiSi}$ зависимости коэффициента Зеебека, электрического сопротивления и теплопроводности обусловлены в основном фазой Гейслера. Для прояснения этого вопроса необходимы тщательные измерения соответствующих свойств примесных фаз $\mathrm{Fe}_{2} \mathrm{Ti}$ и $\mathrm{FeSn}_{2}$ и учет их влияния на термоэлектрические свойства основной матрицы $\mathrm{Fe}_{2} \mathrm{TiSi}$.

\section{4. Заключение}

В работе показано, что теоретические предсказания повышения термоэлектрической добротности $\mathrm{Fe}_{2} \mathrm{TiSn}$ путем частичного замещения $\mathrm{Sn}$ на $\mathrm{Si}$ не подтвердились на практике, что обусловлено формированием многофазного состояния в сплавах $\mathrm{Fe}_{2} \mathrm{TiSn}_{1-x} \mathrm{Si}_{x}(0<x \leq 1)$. Примесные фазы в целом приводят к понижению электрического сопротивления и коэффициента Зеебека и к повышению теплопроводности. На основании наших данных можно сделать предположение, что в окрестности комнатной температуры коэффициент Зеебека в $\mathrm{Fe}_{2} \mathrm{TiSi}$ на порядок меньше, чем в $\mathrm{Fe}_{2} \mathrm{TiSn}$, в то время как электрическое сопротивление и теплопроводность этих соединений близки по своим значениям.

Один из авторов (Д.Ю. Карпенков) благодарен за финансовую поддержку в рамках программы повышения конкурентоспособности НИТУ „МИСиС“ (грант № K4-2015-013). Часть работы выполнена при поддержке совместного исследовательского проекта Института наук о жидкости Университета Тохоку.

\section{Список литературы}

[1] T. Graf, S.S.P. Parkin, C. Felser. IEEE Trans. Magn., 47 (2), 367 (2011).

[2] T. Graf, C. Felser, S.S.P. Parkin. Prog. Solid State Chem., 39, 1 (2011).

[3] Y. Nishino, S. Deguchi, U. Mizutani. Phys. Rev. B, 74 (11), 115115 (2006).

[4] M. Mikami, K. Kobayashi, T. Kawada, K. Kubo, N. Uchiyama. Jpn. J. Appl. Phys., 47, 1512 (2008).

[5] M. Mikami, M. Mizoshiri. J. Electron. Mater., 43, 1922 (2014).

[6] S. Sharma, S.K. Pandey. J. Phys. D: Appl. Phys., 47 (44), 445303 (2014).

[7] D.I. Bilc, G. Hautier, D. Waroquiers, G.-M. Rignanese, P. Ghosez. Phys. Rev. Lett., 114 (13), 136601 (2015).

[8] R.O. Suzuki, T. Kyono. J. Alloys Comp., 377, 38 (2004).

[9] C.S. Lue, Y.-K. Kuo. J. Appl. Phys., 96, 2681 (2004).

[10] A. Slebarski, M.B. Maple, E.J. Freeman, C. Sirvent, D. Tworuszka, M. Orzechowska, A. Wrona, A. Jezierski, S. Chiuzbaian, M. Neumann. Phys. Rev. B, 62, 3296 (2000).

[11] S. Yabuuchi, M. Okamoto, A. Nishide, Y. Kurosaki, J. Hayakawa. Appl. Phys. Express, 6 (2), 025504 (2013).

[12] M. Meinert, M.P. Geisler, J. Schmalhorst, U. Heinzmann, E. Arenholz, W. Hetaba, G. Reiss. Phys. Rev. B, 90 (8), 085127 (2014).

[13] Y. Cai, Y. Wu, Z.Y. Xie, H.S. Liu, Z.P. Jin. Calphad, 49, 110 (2015).

[14] M. Yin, P. Nash, J.A. Kaduk, J.C. Schuster. J. Alloys Comp., 693, 76 (2017).

Редактор А.Н. Смирнов 


\section{Preparation and study of thermoelectric properties of $\mathbf{F e}_{2} \mathrm{TiSn}_{1-x} \mathbf{S i}_{x}$ Heusler alloys}

A.I. Voronin ${ }^{1,2}$, V.Yu. Zueva ${ }^{1}$, D.Yu. Karpenkov ${ }^{1}$, D.O. Moskovskikh ${ }^{1}$, A.P. Novitskii ${ }^{1}$, H. Miki ${ }^{3}$, V.V. Khovaylo ${ }^{1}$

${ }^{1}$ National University of Science and Technology „MISiS“,

119049 Moscow, Russia

${ }^{2}$ National Research South Ural State University, 454080 Chelyabinsk, Russia

${ }^{3}$ Frontier Research Institute for Interdisciplinary Science, Tohoku University,

980-8578 Sendai, Japan

Abstract Experimental investigation of polycrystalline alloys $\mathrm{Fe}_{2} \mathrm{TiSn}_{1-x} \mathrm{Si}_{x}(0 \leq x \leq 1)$ alloys which were theoretically predicted as effective thermoelectric materials was performed. Structural studies showed that partial substitution of Sn for Si results in the formation of a multiphase state in the samples with $x>0$. The impurity phases lead in general to a significant decrease of the Seebeck coefficient and to an increase of the thermal conductivity which does not allow one to consider these materials as perspective thermoelectrics. 